\title{
Manipulation of the resonance interaction in Mach-Zehnder-Fano interferometers
}

\author{
Yi Xu ${ }^{1,2}$ and Andrey E. Miroshnichenko ${ }^{1, *}$ \\ ${ }^{1}$ Nonlinear Physics Centre and Centre for Ultra-high bandwidth Devices for Optical Systems (CUDOS), Australian National University, \\ Canberra ACT 0200, Australia \\ ${ }^{2}$ Laboratory of Photonic Information Technology, School for Information and Optoelectronic Science and Engineering, \\ South China Normal University, Guangzhou 510006, P.R. China
}

(Received 5 May 2011; published 15 September 2011)

\begin{abstract}
We study tunable interaction of the resonances in the Mach-Zehnder-Fano interferometers (MZFIs). A discrete Mach-Zehnder interferometer (MZI) with balanced arms supports bound states in the continuum. We demonstrate that doping an impurity in conventional MZIs gives rise to robust high- $Q$ Fano resonances with asymmetric line shapes. By means of the modified Fano-Anderson model and the scattering-matrix approach, we show that the transmission and the intensity spectra of the whole system are very sensitive to both the location and the strength of the impurity. The side-coupled Fano defects induce an interaction with different eigenmodes of the pure MZI loop. We explore this interaction by tuning the parameters of the Fano defects. The observed resonance interaction can be attributed to the Fano-Feshbach resonance. We further provide with a particular physical example of photonic crystal circuit the applicability of our concept.
\end{abstract}

DOI: 10.1103/PhysRevA.84.033828

PACS number(s): 42.25.Bs, 42.68.Mj, 92.60.Ta

\section{INTRODUCTION}

The Fano resonances manifest themselves as asymmetric profiles in transmission or absorption line shape [1], which originates from the resonant constructive and destructive interference phenomena in wave scattering. During the last decade, the Fano resonances in nanoscale structures have gained numerous attention due to their capability of building sensors, switches, and filters at a very compact scale [2]. As it was discovered by Fano [1], this kind of resonance can be simply regarded as a discrete state interacting with a continuum. By increasing the number of either the discrete states [3] or continua [4] offers more flexibility for engineering the Fano resonances. The conventional Mach-Zehnder interferometer [5] is still popular nowadays due to its powerful capacity of exploring coherent phenomena in physical systems [6,7]. Generally, MZI consists of two coherent continuum paths. Recently, a Mach-Zehnder-Fano interferometer (MZFI) [4] was introduced via coupling a Fano defect to the MZI, which allows for more versatile manipulation of the wave scattering, due to the presence of multiple scattering paths. The Fano resonances are associated with a sharp $\pi$ jump of the scattering phase. The interaction of several Fano resonances results in more sophisticated interference phenomena. Such resonance interaction deserves particular attention since it may result in many phenomena which can be found in various branches of physics, such as, the so-called Fano-Feshbach resonance in nuclear physics [8,9], phase-coherent electron transport in quantum systems [10], analog of electromagnetically induced transparency (EIT) [11-13], coupled-resonator-induced reflection (CRIR) in optical physics [14,15], etc.

The aim of this paper is to demonstrate the possibility of manipulation of resonance interaction in MZFI geometries. For that purpose, we first introduce high- $Q$ Fano resonances of the discrete MZI, which can be considered as bound states

\footnotetext{
*aem124@physics.anu.edu.au
}

in a continuum [16,17] by doping one of the MZI arms with scattering impurities. By analyzing the intensity of the defect sites and the field distribution of the MZI loop, we explore the physical properties of these resonances. Secondly, instead of doping in MZI arms, we side couple a Fano defect to the MZI loop which induces the interaction between different resonant states. Such resonance interaction can be controlled by tuning the eigenfrequency of the side-coupled defects, which will affect the response of the whole structure. It leads to interesting CRIR effect, which is essentially the Fano-Feshbach resonance originating from the strong overlap of two Fano resonances [8,9]. This phenomenon allows us to achieve a tunable intensity of certain nodes of the MZFI via modulating the properties of the side-coupled defect. Among these interesting phenomena, we want to emphasize that the energy exchange between different Fano resonances can be observed at certain eigenfrequency of the Fano defect. Furthermore, we consider the case where two cavities are side coupled to both arms, respectively, with slight different eigenfrequencies. We demonstrate that by designing the coupling symmetry of the corresponding defect-arm subsystems we may realize the analog of EIT. As a particular physical realization of our approach, we study the light propagation in photonic crystals (PhCs), and demonstrate the possibility of the manipulation of Fano resonances in an optical system. Our results open up new opportunities for attractive applications of resonance interactions in photonics and other systems. It should be mentioned that there is related Fano resonance interaction of the Aharonov-Bohm interferometers with two coupled quantum dots in the mesoscopic system [18]. We have to point out that the properties of modes involved in the Fano resonance interaction between Ref. [18] and our present paper are different although the interaction effect is analogous. And both of the resonance interactions can be attributed to the classical Fano-Feshbach resonance [8,9].

The paper is organized as follows. Section II outlines the theoretical model. In Sec. III we discuss the introduction of the high- $Q$ Fano resonance by doping an impurity into one of 


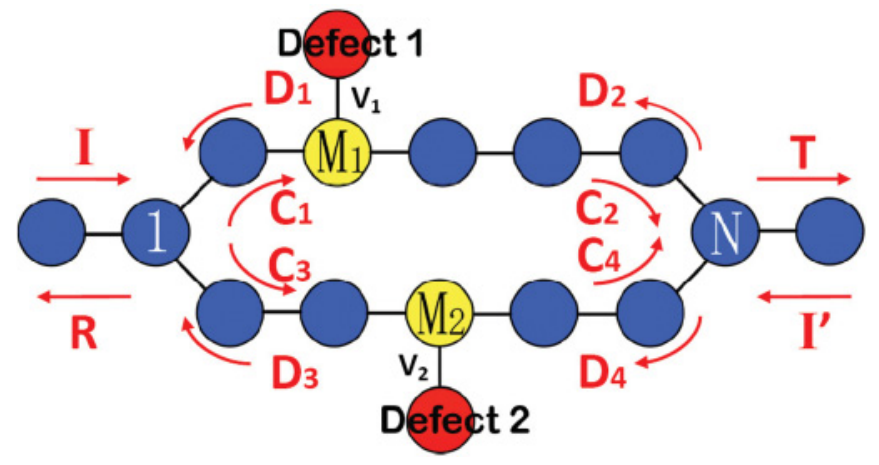

FIG. 1. (Color online) Schematic view of the system (1) describing a general MZFI configuration under consideration. Red sites stand for the side-coupled Fano defects, while the yellow circles represent the on-site impurities in the arms. $M_{1,2}$ correspond to the locations of the scattering potentials. $V_{1,2}$ are the dimensionless coupling strengths between the Fano defects and the loop.

the MZI arms. In Sec. IV, instead of the in-arm impurity, we consider the situation when a Fano defects is side coupled to one of the arms. Section V discusses the role of EIT states in the resonance interactions and Sec. VI provides a particular example of photonic crystal configuration. Section VII concludes the paper.

\section{MODEL}

We study the transmission and the intensity spectra of MZFI configurations based on the modified Fano-Anderson model, which had been successfully used to demonstrate novel Fano resonances in discrete system [3,4,14,19-28]. A conventional MZI with on-site doped defect in one of the arms or side-coupled Fano defects can be described by coupled discrete equations (see Fig. 1) with nearest-neighbour interaction between the discrete sites

$$
\begin{aligned}
i \dot{\psi}_{n} & =\sum_{k} \psi_{k}+\sum_{i=1,2} \delta_{n, M_{j}}\left(\varepsilon_{j} \psi_{M_{j}}+V_{j} \varphi_{d_{j}}\right), \\
i \dot{\varphi}_{d_{j}} & =E_{d_{j}} \varphi_{d_{j}}+V_{i} \psi_{M_{j}},
\end{aligned}
$$

where $\psi_{n}$ represents a complex dimensionless field amplitude excitation of the discrete chain, and we assume the coupling between neighboring sites being equal to unity, $\varphi_{d_{1,2}}$ corresponds to the side-coupled Fano defects, $V_{1,2}$ are the dimensionless coupling strengths between the chain and the Fano defects, $E_{d_{1,2}}$ are the eigenfrequency of the Fano defects, $\varepsilon_{1,2}$ are the strength of the on-site scattering potential, and $M_{1,2}$ are the locations of the scattering potentials in the arm [3]. Due to the limitation of the $Y$ splitter of the MZI loop, there are always forward and backward scattered waves, which carry both amplitude and phase information inside the loop. As the basic component of the MZI, the $Y$ splitter can be modeled with three crossing discrete waveguides at a common point. Note here that the sites forming the junctions are homogeneous while the junctions still behave as a topological defect [28]. Substituting the stationary solutions of the system $\phi_{n} e^{-i \omega t}$ into Eq. (1), the scattering matrix relating the scattering waves in all ports can be found. The transmission and reflection of the system can be obtained by solving the following linear equations with $I=1$ and $I^{\prime}=0$ :

$$
\left(\begin{array}{c}
R \\
C_{1} \\
C_{3}
\end{array}\right)=\mathbf{S}_{L}^{Y}\left(\begin{array}{c}
I \\
D_{1} \\
D_{3}
\end{array}\right), \quad\left(\begin{array}{c}
T \\
D_{2} \\
D_{4}
\end{array}\right)=\mathbf{S}_{-N}^{Y}\left(\begin{array}{c}
I^{\prime} \\
C_{2} \\
C_{4}
\end{array}\right)
$$

with the scattering matrix of the $Y$ splitter expressed as

$$
\mathbf{S}_{K}^{Y}=\left(\begin{array}{lcc}
e^{-2 i K q} r_{Y} & t_{Y} & t_{Y} \\
t_{Y} & e^{2 i K q} r_{Y} & e^{2 i K q} t_{Y} \\
t_{Y} & e^{2 i K q} t_{Y} & e^{2 i K q} r_{Y}
\end{array}\right),
$$

where $K=L,-N$, while $t_{Y}=2 /(3+i \cot q)$ and $r_{Y}=$ $t_{Y}-1$ are the transmission and reflection amplitudes of the $Y$ splitter [28]. The scattering matrix describing the wave interactions at site $M$ with a generalized scattering potential can be written as follows:

$$
\begin{aligned}
\left(\begin{array}{l}
D_{1} \\
C_{2}
\end{array}\right) & =\mathbf{S}_{M_{1}}\left(\begin{array}{l}
C_{1} \\
D_{2}
\end{array}\right), \quad\left(\begin{array}{l}
D_{3} \\
C_{4}
\end{array}\right)=\mathbf{S}_{M_{2}}\left(\begin{array}{c}
C_{3} \\
D_{4}
\end{array}\right), \\
\mathbf{S}_{M_{j}} & =\frac{1}{\tilde{\varepsilon_{j}}-2 i \sin q}\left(\begin{array}{ll}
-\tilde{\varepsilon_{j}} e^{-2 i M_{j} q} & -2 i \sin q \\
-2 i \sin q & -\tilde{\varepsilon_{j}} e^{2 i M_{j} q}
\end{array}\right),
\end{aligned}
$$

where $\varepsilon_{1,2}=\varepsilon_{1,2}+V_{1,2}^{2} /\left(\omega-E_{d_{1,2}}\right)$. The corresponding stationary excitations of each site in the loop can be read as $\left|A_{n}\right|^{2}=\left|C e^{-i q n}+D e^{i q n}\right|^{2}$ except for the sites which induce scattering, $n \neq M_{1,2}, L, N$. Here $C$ and $D$ correspond to the amplitudes of the forward and backward scattering waves, and the site number $n$ should be counted from the site $L(L=1$, without loss of generality). With this model we can study both the transmission and excited intensity spectra of various configurations of the MZFI structures.

\section{MZI RESONANCES INDUCED BY DOPED ON-SITE IMPURITIES}

In the case of a perfect MZI structure without any impurities the waves propagating in both arms are in phase and the response of the system (1) exhibits perfect transmissions at certain resonance frequencies. Figure 2(a) presents the transmission of the pure MZI with the dispersion $\omega=2 \cos q$. The total number of resonances corresponds to the number of standing waves that can be supported by the loop, which is proportional to the length of the arms $|L-N|$. In Fig. 2(b) we plot the distribution of the wave intensity at perfect transmission frequencies for the case $|L-N|=6$. Such field distributions correspond to the excitation of standing waves, and can be attributed to effective Fabry-Perot resonances. Note here, that there is a symmetry in the field distributions with respect to the wave number $q$ around the propagation band center.

With the harmonic assumption, we obtain a set of linear equations with eigenvector $\phi_{n}$ and eigenvalue $\omega_{n}$ describing the balanced MZI. Such a system can be solved by using periodic boundary condition. As can be seen from Fig. 3, the spectrum resembles the continuum spectrum of an infinite discrete chain with the dispersion $\omega=2 \cos q$ except for four bound states outside the continuum and five embedded states inside the continuum (see Fig. 3). By substituting the standing wave solution $A e^{i q n}-A e^{-i q n}$ and choosing one of the junction sites as the phase reference, we can obtain the 


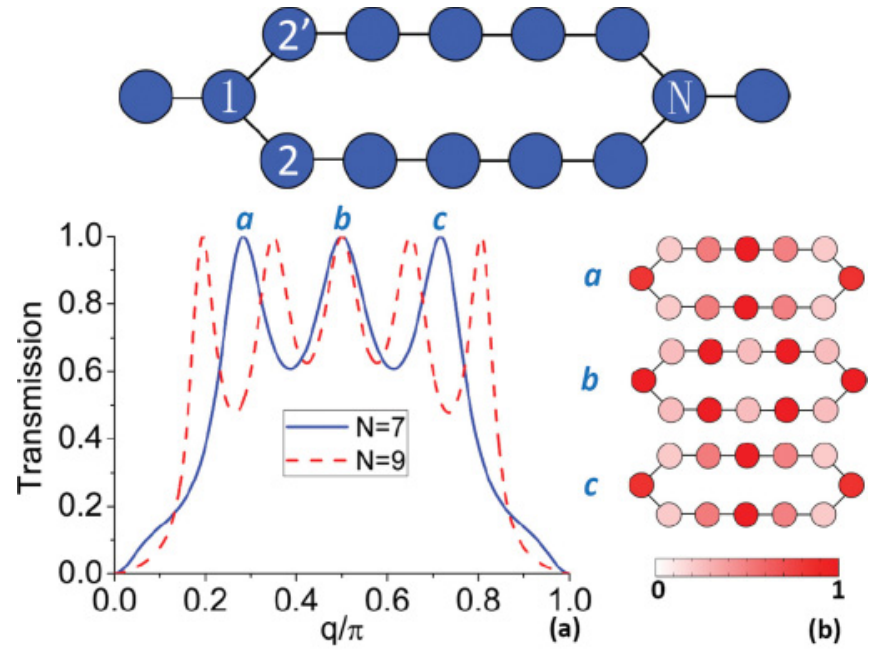

FIG. 2. (Color online) Comparison of the scattering properties of two pure MZI structures without any defects $N=7$ (solid blue) and $N=9$ (dashed red): (a) transmission and (b) intensity of the MZI loop at different perfect transmission points marked at plot (b).

frequencies of the embedded states as $\pm \sqrt{3}, \pm 1,0$, when $L=1, N=7$, and $A$ is a complex number. The corresponding field distribution of marked states are shown in the insets. Note that we are working with real matrix and the white color means no excitation. From the field distribution of the bound states $1^{\prime}-4^{\prime}$, one can find that the fields concentrate at the junction sites and exponentially decay (data are not show here). It means that the junctions behave as defects and this is the key reason to form the local states outside the band. We can also see from Fig. 4 that the MZI with balanced arms supports "dark states" $1-5$. Such dark states are standing waves forming in each arm, and the corresponding sites next to the junction (i.e., site 2 and site $2^{\prime}$, site 6 and site $6^{\prime}$ ) are out of phase. It is interesting to
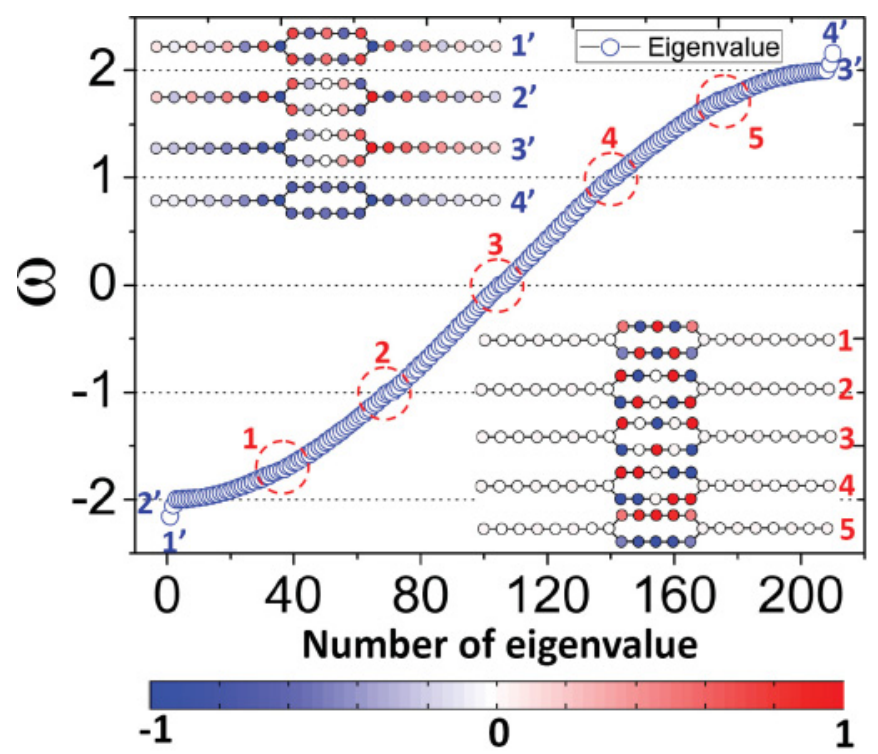

FIG. 3. (Color online) Eigenvalue of the balanced MZI when $L=1, N=7.1^{\prime}-4^{\prime}$ mark the bound states outside the continuum while 1-5 mark the embedded states in the continuum. The insets show the field distribution of the corresponding mode. (a)
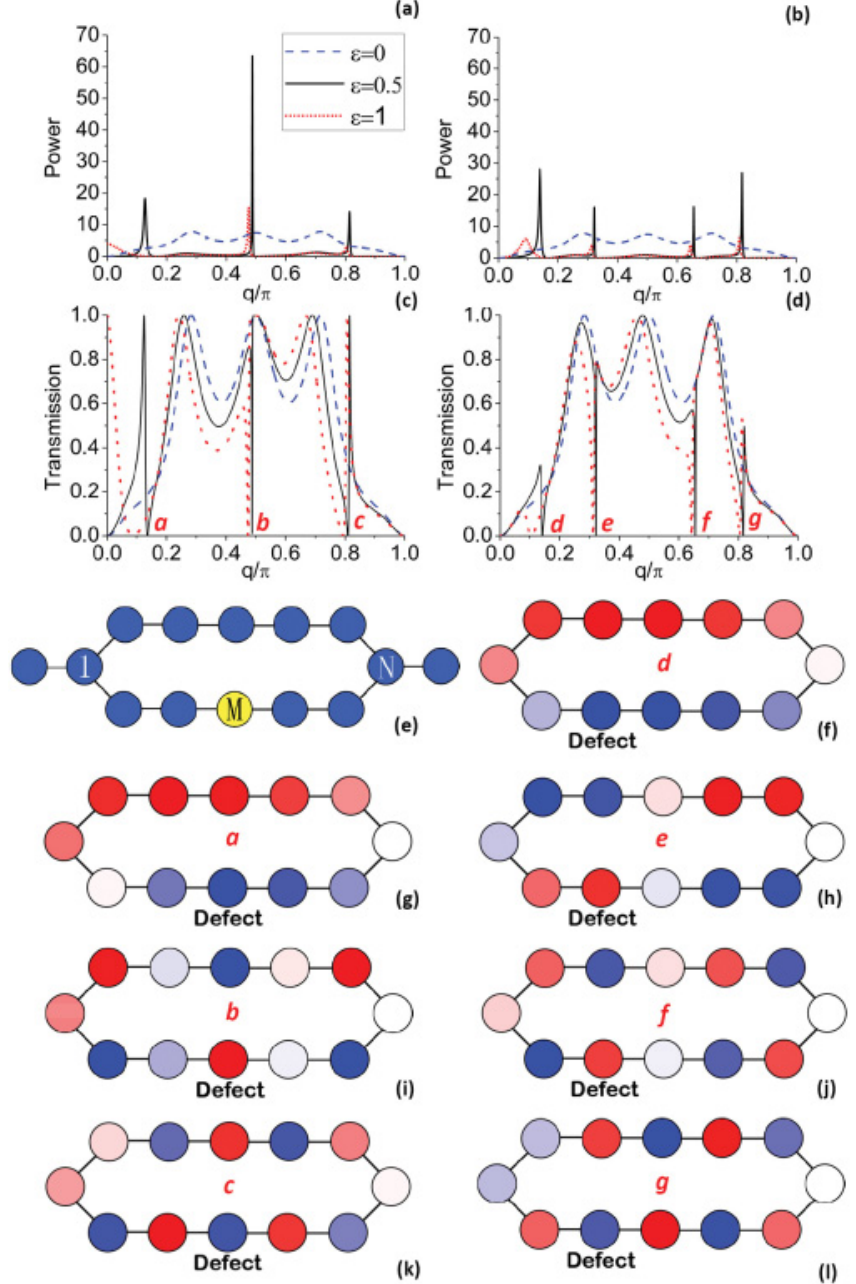

$-1$

(k)

(I)

FIG. 4. (Color online) Scattering properties of MZI configurations with doped on-site defect in one of the arms located symmetrically at $M=4[(\mathrm{a})$ and (c)] and asymmetrically at $M=3$ [(b) and (d)], respectively, with $L=1, N=7$. Plots (a) and (b) show the intensity of the defect site with different on-site impurity strengths and locations. Plots (c) and (d) show the transmission of the whole structure. Dashed blue lines show the results of the pure MZI structure for comparison. The solid blue lines and the dotted red lines represent the situation when the doped impurity strength is $\varepsilon=0.5$ and $\varepsilon=1$, respectively. (a)-(d) share the same line style. (e) Sketch map of the doped MZI. (f)-(l) Field distribution (real part of $A_{n}$ ) of the MZI. They correspond to certain wave numbers marked a-g in (c) and (d). All of them are normalized to the maximum of each particular curve, indicated by a color bar. The location of the defect is indicated by the word.

find that such simple structure supports embedded states in the continuum $[16,17]$. However, such embedded states cannot be excited by the scattering wave from one of the inputs with fundamental even mode due to the mode symmetry mismatch. If we break the symmetry of the system, for example, by doping an impurity in one of the arms, we have the opportunity to access the embedded states. With the impurity located at the the node (referring to the insets $1-5$ of Fig. 3), we cannot excite the corresponding embedded states. Nevertheless, if the 
impurity is placed at a certain site with a nonzero excitation shown by the inset $1-5$ of Fig. 3, specified sets of embedded states can be targeted. Therefore, one can choose which set of the embedded states to be excited.

Doping the arms of MZI with an on-site impurity [as shown in Fig. 4(e)] will result in drastic variation of the scattering amplitudes and phases. In particular, it will lead to the formation of a discrete state (embedded-like state) inside the continuum of propagating waves, responsible for the appearance of the Fano resonances in the transmission. Note, that the intensity of the defect site including the junction can be obtained via

$$
\left|A_{d}\right|^{2}=\left|\frac{\left(\Sigma^{k} A_{k}\right)}{\omega-\varepsilon}\right|^{2},
$$

where $k$ stands for the number of neighbor sites. The denominator in Eq. (5) implies that the intensity of the defect site is related to the frequency of the scattering wave and the strength of the impurity. The intensity spectra of the defect site with two typical doped impurities in the arms at $M=4$ and $M=3$ sites are presented in Figs. 4(a) and 4(b), respectively. It shows two combinations of resonances due to different types of doping symmetry. The total intensity of the pure MZI loop is plotted by dashed blue lines for a reference. We can identify that the defect site is highly excited at certain wave numbers, which corresponds to resonant scattering conditions. It can be further confirmed by the results in Figs. 4(c) and 4(d) that some robust resonant reflections are caused by the impurity doping. Depending on the coupling symmetry, one can choose to excite certain resonances between these two sets. If the impurity is placed at the site next to the junction in the arm, all five embedded states can be excited. The shift of resonant frequency referring to the eigenbound states in the continuum is related by the strength of the impurity as can be seen from Figs. 4(a)-4(d). It is more straightforward to inspect the field distribution and the phase at different sites. Figures 4(f)-4(1) show the real part of the $A_{n}$ when $\varepsilon=0.5$. Each field distribution is denoted by the marked resonances in Figs. 4(c) and 4(d). Indeed, we can find that all of the resonances resemble the corresponding embedded states (modes 1-5) plotted in Fig. 3. Such modes are the standing waves formed in two arms. Therefore, the transmission is blocked, and a very large $Q$ factor can be anticipated due to the mode symmetry mismatch [29]. At the same time, the total intensity in the arm builds up due to the freezing of the waves. These kinds of resonances can be attributed to the Fano resonance with the interference between a continuum spectrum (the original pure MZI) and a discrete state (localized mode in the loop created by the impurity). According to general properties of Fano resonances, the phase of the scattering wave acquires a $\pi$ shift in the vicinity of the resonance. The original in-phase waves propagating in both arms are perturbed to be out of phase at the input and the output ports leading to the sharp and asymmetric line shape [30]. One can also see from Figs. 4(a) and 4(b) that the smaller impurity strength corresponds to the higher excitation of the defect site because of the little amount of odd modes being excited. We have to point out that resonances at the band edge always have a smaller $Q$ factor than the ones near the band center. This

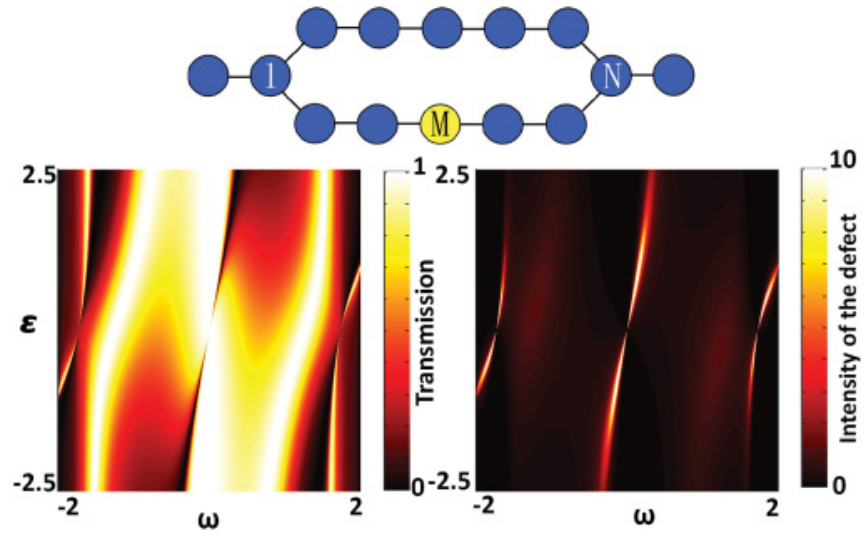

FIG. 5. (Color online) (a) Transmission and (b) intensity spectra of the defect site for symmetrically placed impurity vs frequency $\omega$ and impurity strength $\varepsilon$.

phenomenon can be explained by the different overlap between the continuum and the discrete modes [26,27].

To gain a full picture of the interaction, we calculate the transmission and the intensity spectra of the defect site by varying the scattering potential strength from $\varepsilon=-2.5$ to $\varepsilon=2.5$. Figure 5 shows the evolution for impurity located symmetrically at site $M=4$. The asymmetric case with $M=3$ is similar and omitted. Note here, that the colorscale of the excitation in this paper is saturated to increase the better readability. When $\varepsilon$ is zero only the Fabry-Perot resonances of the pure MZI can be excited. For nonzero impurity strength $\varepsilon \neq 0$, one may expect the appearance of the transmission resonances associated with the excitation of induced localized modes. It should be pointed out that the scattering phase $\left[\arg \left(\frac{-2 i \sin q}{\varepsilon-2 i \sin q}\right)\right]$ in the arm with the impurity are conjugated for opposite values of the impurity strength $\pm \varepsilon$ at a given frequency. It can be seen from Fig. 5 that the frequency of the MZI loop's resonances would shift monotonously along the band with the impurity strength $\varepsilon$. The larger the absolute value of scattering potential, the bigger the shift from its original position. Different on-site energies correspond to different scattering phase shifts of the propagating waves, which in turn shift the resonance frequencies.

\section{TUNANBLE INTERACTION OF MZI AND FANO RESONANCES}

As it was shown in the previous section, changing the energy of the on-site impurity will imply the external tunability of the transmission resonances. We would like to take this approach one step further and consider side-coupled Fano defect attached to the MZI loop. By coupling a cavity with local energy value $E_{d}$ to the propagation chain, one may expect the appearance of the Fano resonance at the frequency $\omega=E_{d}$ [23]. Generally, Fano resonance of the side-coupled defect induces a $\pi$-phase shift to the scattering waves in the vicinity of resonance frequency. Therefore, the waves passing the continuum path (MZI loop) and the discrete path (Fano defect) will suffer from destructive interference, that is, resonance reflection. When a Fano defect is coupled to the MZI loop, one of the coherent arms provides an additional continuum path for the scattering wave to propagate, which endows the 
system with more abundant and interesting interference. As it is known, Fano defect can be considered as a resonant scattering potential with a frequency dependent strength as $\varepsilon^{\prime}=V^{2} /\left(\omega-E_{d}\right)$. As a result, the Fano defect acts as a generalized doped impurity with tunable strength. However, the scattering phase conjugation is more tricky, which is not only related to the energy of the defect, but also the frequency as well. Such a relationship is the key reason to producing bifurcation in the intensity-spectra map, which will be addressed in the following. The excitation of the Fano defect can be obtained as follows:

$$
|B|^{2}=\left|\frac{V\left(\Sigma^{k} A_{k}\right)}{\left(\omega-\varepsilon^{\prime}\right)\left(\omega-E_{d}\right)}\right|^{2} .
$$

The numerator implies the possibility that the Fano defect can be highly excited not only at its eigenfrequency, that $i s, \omega \neq E_{d}$, but at the frequency of the loop's resonance as well, when the site coupled to the Fano defect $A_{M}$ is highly excited (according to Sec. II it works like an effective scattering impurity in the arm). It means that the side-coupled Fano defect can excite the embedded states of the MZI as well. As a result, the intensity of the Fano defect builds up mediated by the coupled site $M$. It is very interesting to note that both MZI loop and the Fano defect are highly excited at such resonances. This is completely different to the situation when the Fano defect is highly excited due to a vanishing denominator in Eq. (6) at the eigenfrequency of the Fano defect, where the coupled site $M$ does not have to be excited [23]. Figure 6 shows the transmission of the system and the intensity of the Fano defect with different coupling strength $V$. The eigenfrequency of the Fano defect is $\omega=E_{d}$ and it is side coupled to the site $M=3$, which is indicated by the inset in Fig. 6. One can see that both

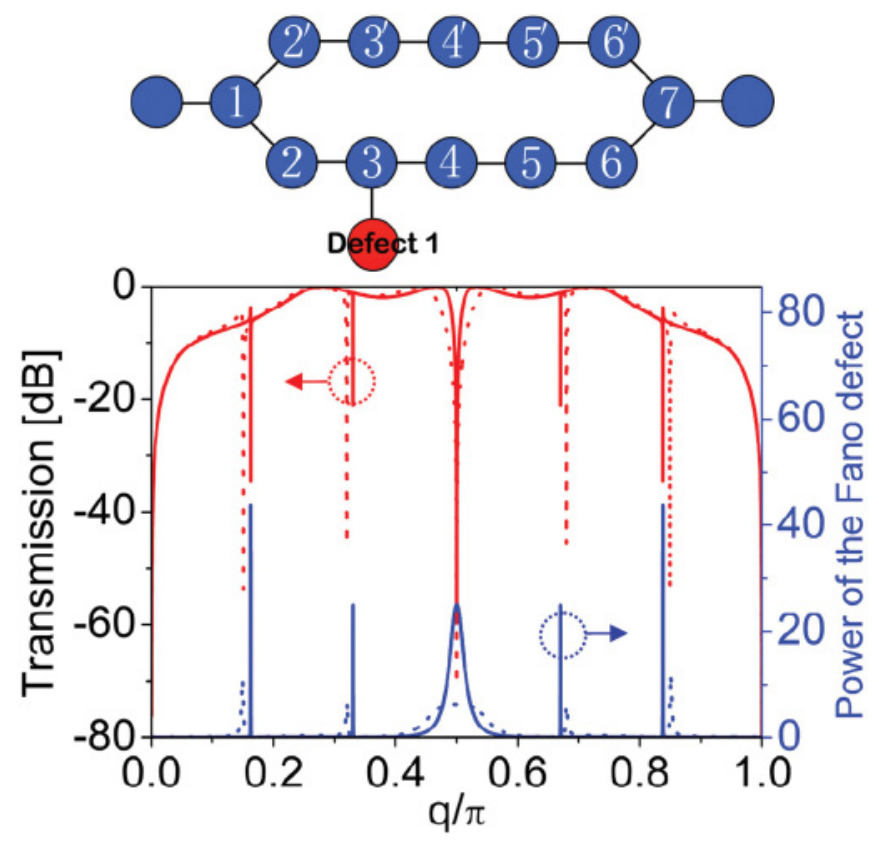

FIG. 6. (Color online) Transmission and intensity spectra of the MZFI structures for different coupling strengths $V$. Solid lines stand for $V=0.4$ while dotted lines represent $V=0.8$. The eigenfrequency of the Fano defect is $E_{d}=0$, which corresponds to the wave number $q=\frac{\pi}{2}$. the strength and the position of the MZI's resonance are related to the scattering properties of the Fano defect, which makes the resonance of the whole loop tunable via the side-coupled defect only. The interaction of Fano defect with the MZI loop provides another degree of freedom for the scattering wave to interfere and gives birth to a type of Fano resonances.

According to the above discussion, the resonances of the MZFI are related to the side-coupled Fano defect. By varying the eigenfrequency of the Fano defect $E_{d}$ along the propagation band, the strong interaction between eigenmodes of MZI and side-coupled Fano defect can be expected. Figure 7 shows the transmission, the sum of intensity in the loop, and the intensity of symmetrically and asymmetrically placed Fano defect. The eigenfrequencies of the Fano defect span in the region $E_{d}=-2.5$ to $E_{d}=2.5$ as indicated by the dashed lines. All other parameters can be found in the caption. As it can be seen from Fig. 7, the response of the system depends on both the location and the eigenfrequency of the side-couple cavity. The MZI loop's total intensity strength varies with the
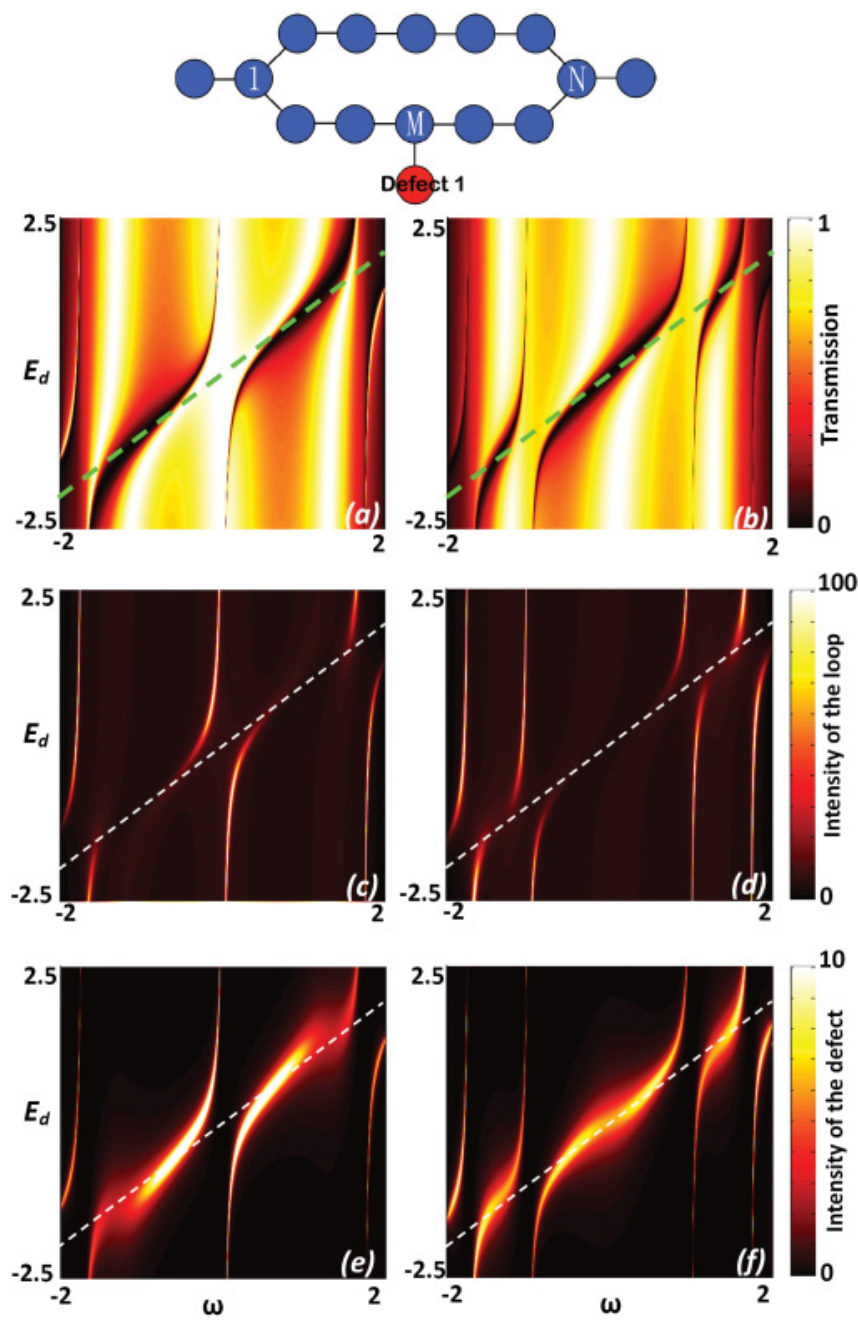

FIG. 7. (Color online) (a) and (b) Transmission, (c) and (d) the sum of the intensity in the MZI loop, and (e) and (f) the intensity of the Fano defect vs input frequency and eigenfrequency of the Fano defect for (a), (c), and (e) symmetric $M=4$ and (b), (d), and (f) asymmetric $M=3$ side-coupled defect. In all the cases, $L=1$, $N=7$, and $V=0.8$. Inset shows the schematic view of the MZFI. 
shift of $E_{d}$ according to the strength of the effective scattering potential [as in Figs. 4(a)-4(d)]. At the same time, the mode transformation and energy exchange take place where a hybrid Fano resonance appears. Such transformation is more clearly observed in Figs. 7(e) and 7(f). The resonant Fano eigenmode is transformed to a hybrid Fano resonant mode with two sharp resonances together with the varying of $E_{d}$. The complete transformation is exactly at the bifurcation point $\left(E_{d}=0\right)$ where the dispersion is minimized [see Figs. 7(c)-7(f)]. The scattering potential diverges at the eigenfrequency of the Fano defect and becomes complex conjugated at either side of the eigenfrequency. Due to the complex conjugation of the scattering phase in the doped arm and the mutual dependence between two kinds of resonances, $E_{d}$ would not coincide with the resonance frequency of the loop. When both resonances are getting closer, the phase conjugation starts to affect strongly MZI loop's resonances and gradually transfers its energy from one mode to another. As a result, drastic variation of the scattering phase produces two narrow resonances when the resonant frequency difference between the Fano eigenmode and the MZI loop's resonance comes to the minimum. We also notice that the total intensity of the MZI in the vicinity of this bifurcation is maximally reduced which can be confirmed by Figs. 7(c) and 7(d). It means that the energy transformation is mediated by the Fano defect as it is demonstrated in Figs. 7(e) and 7(f). When $E_{d}$ is far away from the MZI loop's resonances, the interaction between these two modes becomes negligible. The blurred regions appearing in Figs. 7(e) and 7(f) are the corresponding signature of the eigen-Fano resonance. All of these suggest the possibility of switching between the eigenand hybrid-Fano resonant modes. When $E_{d}$ moves out of the propagation band, the eigenresonance of the Fano defect cannot interact with the loop's resonance inside the continuum. Thus the side-coupled Fano defect works like a scattering impurity in the arms and the resonances resemble the one in Fig. 5. Thus, we found the resonance interaction between the MZI loop's resonance and the single side-coupled Fano defect.

As a next step, we explore the interaction of MZI resonances with two coupled Fano defects with detuned eigenfrequencies $E_{d_{1}}=E-0.1$ and $E_{d_{2}}=E+0.1$ attached symmetrically and asymmetrically, respectively (see Fig. 8). Both sets of even and odd resonant modes of the MZI loop are excited at the same time in such a structure (compare to Fig. 4). The intensity map of the Fano defect 1 is similar to the case of Fig. 7(f), while the one of the Fano defect 2 is analogous to Fig. 7(e) due to coupling symmetry. The discrepancy between Figs. 8(c) and 7(f) as well as Figs. 8(d) and 7(e) is due to the presence of two Fano defects. It should be pointed out that the interaction between the Fano defects and the MZI loop's resonances near the band edge is weak because of the reduced strength of resonances. That is why only three clear distinguished interactions can be obtained in Figs. 7 and 8 .

The key result of our study is that the resonance interaction can be understood by means of a very general physical mechanism associated with the Fano-Feshbach resonances $[8,9]$. In the resonance overlapping regime, where the
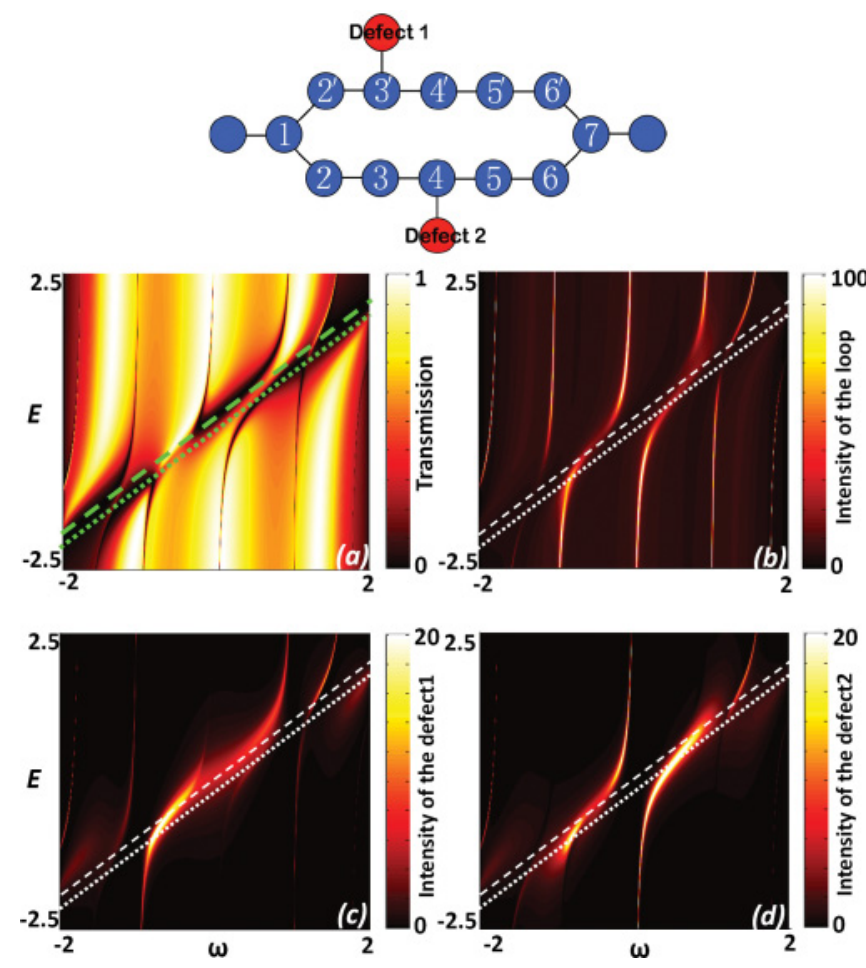

FIG. 8. (Color online) (a) Transmission, (b) and (c) the sum of the intensity in the MZI loop, and (c) and (d) the intensity of two Fano defects vs input frequency $\omega$ and the central frequency $E$ of Fano defects with $E_{d_{1}}=E-0.1$ and $E_{d_{2}}=E+0.1$ for the MZFI structure shown above. The coupling strengths of both defects are identical $V_{1,2}=0.8$.
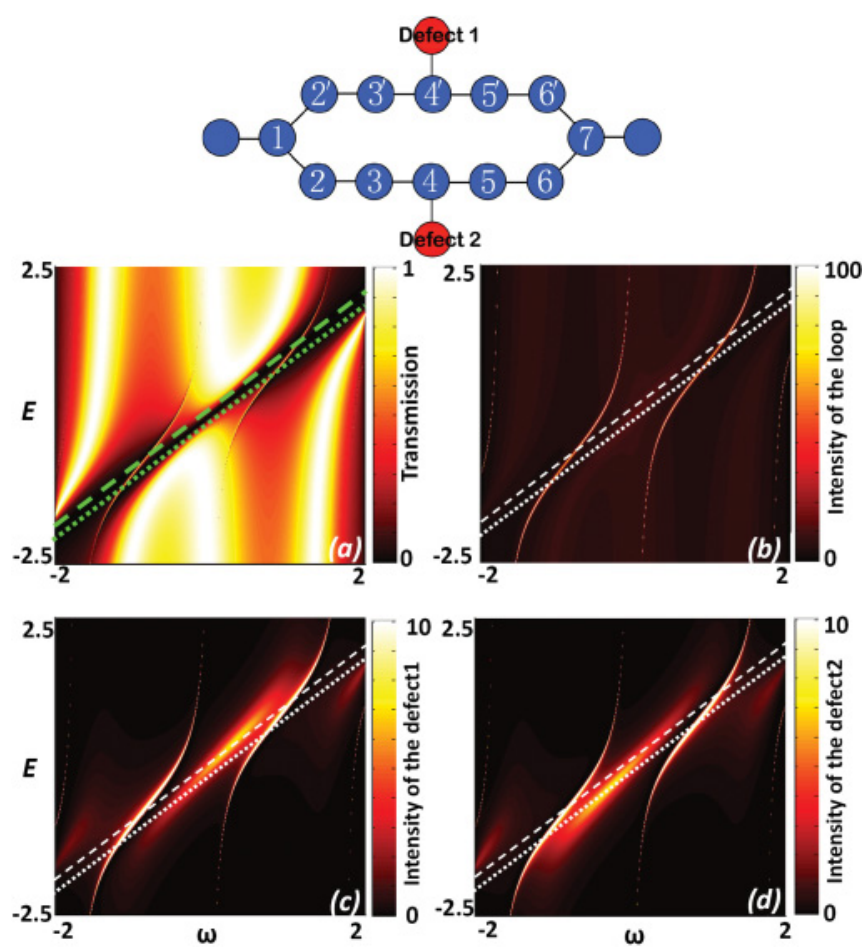

FIG. 9. (Color online) (a) Transmission, (b) the sum of the intensity in the MZI loop, and (c) and (d) the intensity of two symmetrically coupled Fano defects vs input frequency $\omega$ and the central frequency $E$ of Fano defects with $E_{d_{1}}=E-0.1$ and $E_{d_{2}}=E+0.1$ for the MZFI structure shown above. The coupling strengths of both defects are identical $V_{1,2}=0.8$. 

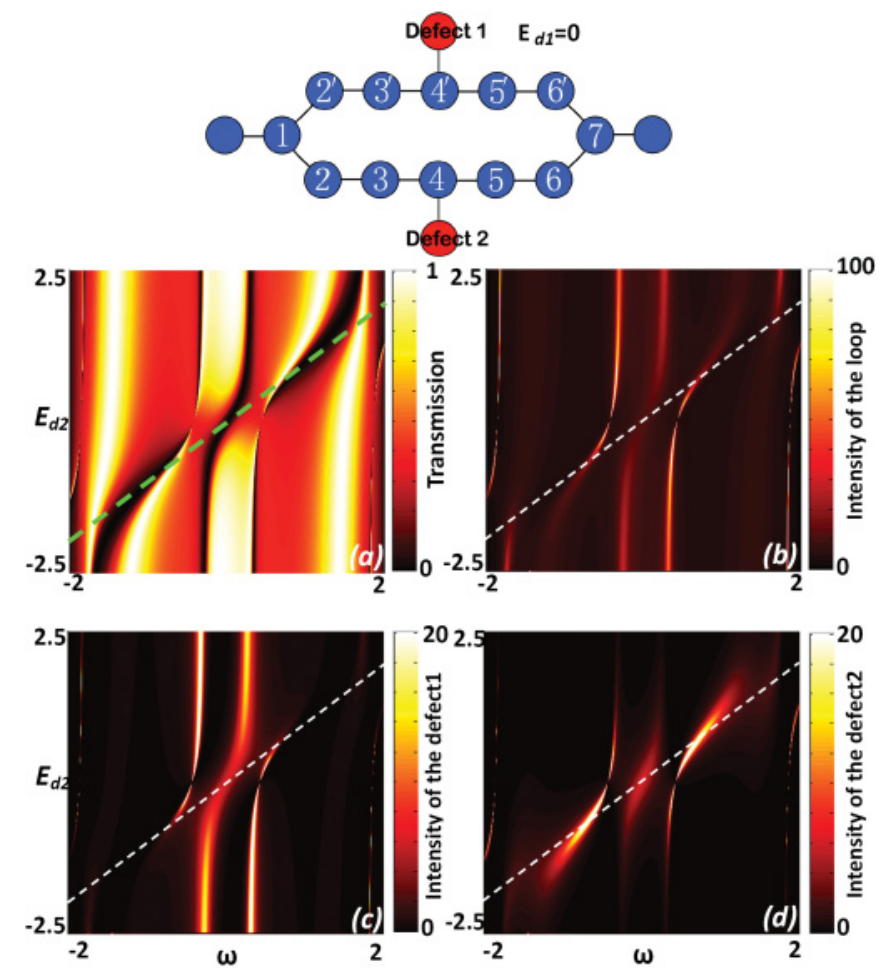

FIG. 10. (Color online) (a) Transmission, (b) the sum of the intensity in the MZI loop, and (c) and (d) the intensity of two symmetrically coupled Fano defects vs input frequency $\omega$ and the eigenfrequency of the second Fano defect $E_{d_{2}}$, while the eigengrequency of the another Fano defects is fixed $E_{d_{1}}=0$ for the MZFI structure shown above. The coupling strengths of both defects are identical $V_{1,2}=0.8$.

frequency separation between the Fano and MZI resonances is comparable or smaller than their spectral widths, one may expect the emergence of ultra-narrow high- $Q$ resonance due to sharp phase modulation of two interacting resonances. Such complex scattering phase deformation is accompanied with the energy transfer between interacting modes, which offers an opportunity for external control.

\section{MANIPULATION OF EIT-LIKE STATES}

A particular example of the interacting Fano resonances is the scattering response which mimics the EIT phenomenon found in three-level atoms. In the previous section we varied the central frequencies of both Fano defects keeping the difference between them constant $\left|E_{d_{1}}-E_{d_{2}}\right|=$ const, and no EIT-like effects were observed. In order to fully resemble the EIT condition of quantum interference in the atom structure, two slightly different Fano defects should be placed with the same coupling symmetry to obtain two effective indistinguishable and coherent paths which connects the common state [31]. Figure 9 presents the evolution of the complex interaction of the Fano resonances when two defects are coupled symmetrically at sites $M_{1,2}=4$. The case of asymmetric coupling $M_{1,2}=3$ is similar and not presented here. As can be seen from Fig. 9, the EIT-like state appears when the eigenfrequencies of the Fano defect are away from the MZI loop's resonances. It is interesting to note that two very sharp resonances appear together with a resonant reflection in
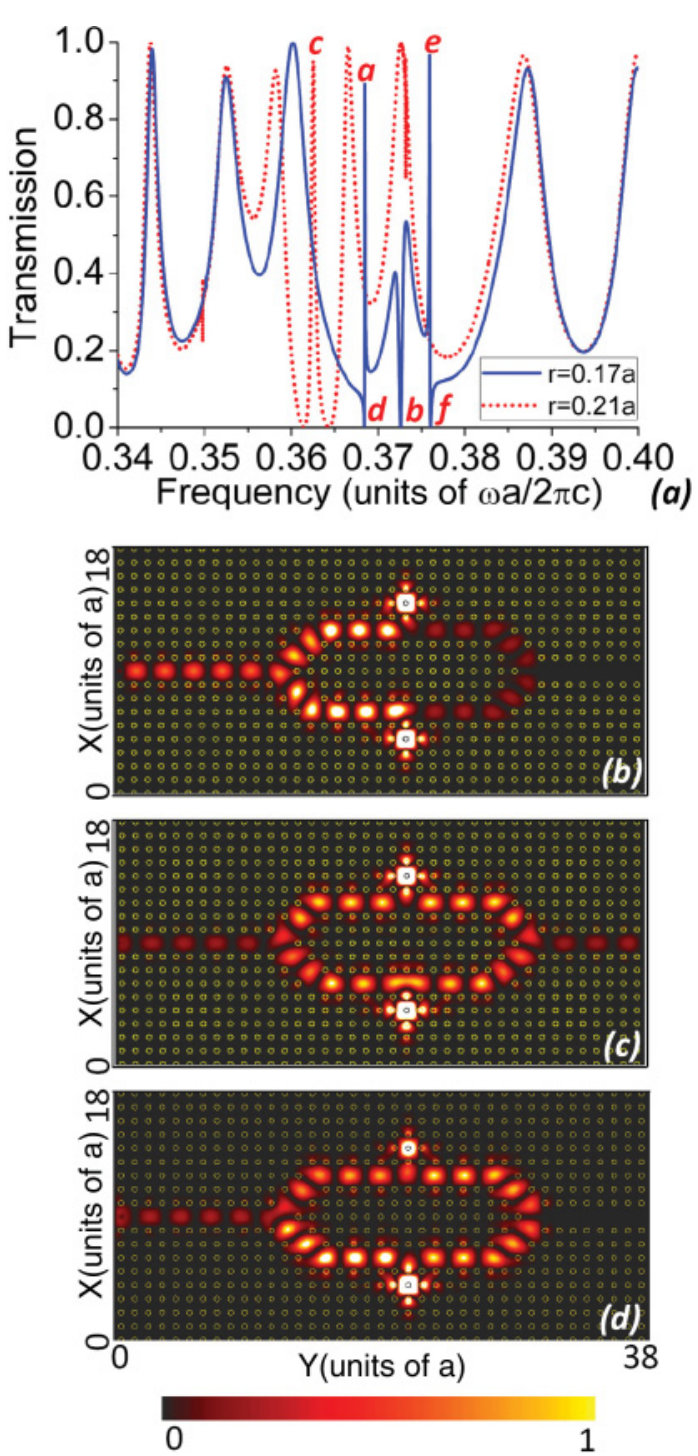

FIG. 11. (Color online) Numerical simulations of 2D PhC realization of MZFI structure. (a) Transmission with two slightly different side-couple Fano defects. The solid blue line stands for the case when $r=0.17 a$ while the dotted red line represents $r=0.21 a$, where $a$ is the lattice constant. (b), (c), and (d) are the corresponding electrical field distributions $|E|^{2}$ at the resonances marked in (a). The FDTD grid is nonuniform. The grid size near the defect is $a / 100$ to tell the small difference in radius while the others is $a / 50$.

between. We notice that these sharp resonances correspond to high intensity of both the Fano defects and the MZI loop, which resemble the hybrid Fano resonant modes presented in the previous section. The same mode transformation is still preserved while now it is between the EIT-like mode and the hybrid Fano resonant modes. As can be seen from Fig. 2(d) in Ref. [15], the EIT state is always with two successive $\pi$ jump of the scattering phase in the vicinity of the total transmission. The MZI loop's resonances perturb one of two successive phase jumps which eventually convert it into a destructive interference in between two sharp and asymmetric resonances.

For completeness, we present the situation when the eigenfrequency of one the Fano defects is fixed $\left(E_{d_{1}}=0\right)$ while the other one is varying. Figure 10 shows the results 
for symmetric coupling with $M_{1,2}=4$. When the difference between the eigenfrequencies of two defects is large, the results are similar to the case with only one Fano defect coupled to the MZI. The situation changes when the eigenfrequency of second defect is near the MZI loop's resonances, two Fano defects start to interact and produce two successive phase jumps. Such interaction produces two hybrid Fano states and the corresponding resonant reflection in between as shown in Fig. 9. The hybrid mode transformation is more clear in Fig. 10(c). The intricate interference of more than two Fano resonances can be again attributed to the Fano-Feshbach resonance [8,9], which comprises of couple resonance induce transparency and CRIR at the same time. Further increasing the numbers of the side-coupled Fano defect would bring more fruitful interference to the system.

\section{PHOTONIC CRYSTAL MZFI CIRCUIT}

Based on the above analysis, one can expect that by coupling Fano defects to MZI and tuning their parameters it is possible to manipulate resonance interaction between Fano and MZI modes, which results in tunable transmission and excited intensity of the whole system. Note that the model considered above is quite general and can be applied to different physical systems. For our purposes, we take the two-dimensional $\mathrm{PhCs}$ as an example to demonstrate the existence and the manipulation of the Fano resonance, which can be mapped with the help of Green's function formalism to discrete systems [32]. The PhCs consists of dielectric rods arranging in a square lattice. And air is the background. The radius $r=0.19 a$, where $a$ is the lattice constant and the refractive index $n$ is equal to 3.14. This kind of PhCs has a complete band gap within the frequency range $0.316-0.4452 \pi a / c$ of the transverse-electric waves (magnetic-field parallel to the plane). The MZI is made by removing certain rods while the side-couple Fano defect is made by replacing one rod which is $2 a$ away from the arm with a polymer $\operatorname{rod}(n=1.63)$. Such a defect supports a quadrupole mode at $f \approx 0.36982 \pi c / a$ as show in Figs. 11(b)-11(d). In order to produce two slightly different Fano defects, we impose a radius difference $\left|r_{d_{1}}-r_{d_{2}}\right|=0.01 a$ between two defect rods. The finite-difference time-domain (FDTD) method is employed to demonstrate such Fano resonance manipulation. Figure 11(a) shows the total transmission of the system. Other numerical parameters can be found in the caption. The solid blue line presents the situation that the EIT state interacts with the resonance of the MZI. As can be seen from Fig. 11, two hybrid sharp and asymmetric resonances appear while one of the resonant reflection still persist. The dotted red line stands for the EIT state moving away from the resonance of the MZI loop. The specified electric field distributions are shown in Figs. 11(b)-11(d). Each of them corresponds to the marked resonances in Fig. 11(a). Please note that the electric intensity distribution of the mark resonances $a, c$, and $e$ are similar although there are different energy concentration ratios in two defects and certain sites of the arms and, thus, we show only one of them $c$ for convenience. It can be seen from the electric intensity distribution that distinct wave interference takes place at three different types of resonances. The electric intensity distribution of $b$ presents the prominent characteristic of resonant reflection by two defects [see Fig. 11(b)]. The EIT state $c$ and the tip of the hybrid resonances ( $a$ and $e$ ) share the properties of highly enhanced electric intensity of both the MZI loop and two defects as well as total transmission at the output port [see Fig. 11(c)]. While the electric intensity distribution of the dips of the hybrid resonances ( $d$ and $f$ ) and $a, c$, and $e$ are similar except for resonant reflection [see Fig. 11(d)]. These numerical experiments are consistent with the theoretical results [Figs. 9(a)-9(d)], and indicate future application of the manipulation of Fano resonances.

\section{CONCLUSIONS}

Combing the generalized discrete Fano-Anderson model and the scattering matrix method, we studied the transmission and the intensity spectra of the doped MZI structures. We found that by doping impurity into a common MZI, certain robust Fano resonances would arise. The use of the sidecoupled Fano defect as the impurity makes the scattering more sophisticated. We demonstrated the possibility of tunable resonance interaction and modes transformation between different Fano and MZI loop's resonances. By further increasing the number of side-coupled defects, we effectively increased the degrees of freedom for the propagating wave resulting in fine manipulation of the scattering properties. Particularly, we presented the situation where the EIT-like states interact with the MZI resonances, which could result in distinct hybrid sharp asymmetric resonance together with total resonant reflection. We demonstrated that such interesting phenomena can be understood in terms of the general physical mechanism of the Fano-Feshbach resonance, which describes the complex interference between overlapping several Fano resonances.

We anticipate that the proposal of manipulating the Fano resonances can find its applications in a variety of different linear and nonlinear physical systems. Such as enhancing biosensor, fluorescence detection, linear and nonlinear switching, etc.

\section{ACKNOWLEDGMENTS}

The authors thank Professor Yuri Kivshar and Dr. Anton Desyatnikov for useful discussions. Andrey Miroshnichenko was supported by the Australian Research Council. Yi Xu acknowledges the support from the China Scholarship Council and the Nonlinear Research Centre at ANU for their hospitality.
[1] U. Fano, Phys. Rev. 124, 1866 (1961).

[2] A. E. Miroshnichenko, S. Flach, and Yu. S. Kivshar, Rev. Mod. Phys. 82, 2257 (2010).

[3] A. E. Miroshnichenko and Yu. S. Kivshar, Phys. Rev. E 72, 056611 (2005).
[4] A. E. Miroshnichenko and Yu. S. Kivshar, Appl. Phys. Lett. 95, 121109 (2009).

[5] E. Mach, The Principles of Physical Optics (Dover, New York, 2009), p. 170 (first published in German in 1926). 
[6] Y. Ji, Y. Chung, D. Sprinzak, M. Heiblum, D. Mahalu, and H. Shtrikman, Nature (London) 422, 415 (2003).

[7] M. Born and E. Wolf, Principles of Optics, 7th ed. (Cambridge University Press, Cambridge, 1999).

[8] H. Feshbach, Ann. Phys. (NY) 5, 357 (1958).

[9] H. Feshbach, Ann. Phys. (NY) 19, 287 (1962).

[10] O. Valsson, C.-S. Tang, and V. Gudmundsson, Phys. Rev. B 78, 165318 (2008).

[11] D. D. Smith, H. Chang, K. A. Fuller, A. T. Rosenberger, and R. W. Boyd, Phys. Rev. A 69, 063804 (2004).

[12] Q. Xu, S. Sandhu, M. L. Povinelli, J. Shakya, S. Fan, and M. Lipson, Phys. Rev. Lett. 96, 123901 (2006).

[13] R. W. Boyd and D. J. Gauthier, Nature (London) 441, 701 (2006).

[14] S. F. Mingaleev, A. E. Miroshnichenko, and Yu. S. Kivshar, Opt. Express 16, 11647 (2008).

[15] A. E. Miroshnichenko, Phys. Rev. E 79, 026611 (2009).

[16] H. Friedrich and D. Wintgen, Phys. Rev. A 31, 3964 (1985).

[17] H. Friedrich and D. Wintgen, Phys. Rev. A 32, 3231 (1985).

[18] V. Moldoveanu, M. Ţolea, A. Aldea, and B. Tanatar, Phys. Rev. B 71, 125338 (2005).

[19] S. Noda, A. Chutinan, and M. Imada, Nature (London) 407, 608 (2000).

[20] S. Fan and J. D. Joannopoulos, Phys. Rev. B 65, 235112 (2002).
[21] S. Fan, W. Suh, and J. D. Joannopoulos, J. Opt. Soc. Am. A 20, 569 (2003).

[22] S. Flach, V. Fleurov, A. V. Gorbach, and A. E. Miroshnichenko, Phys. Rev. Lett. 95, 023901 (2005).

[23] A. E. Miroshnichenko, S. F. Mingaleev, S. Flach, and Yu. S. Kivshar, Phys. Rev. E 71, 036626 (2005).

[24] A. E. Miroshnichenko and Yu. S. Kivshar, Opt. Express 13, 3969 (2005).

[25] S. F. Mingaleev, A. E. Miroshnichenko, Yu. S. Kivshar, and K. Busch, Phys. Rev. E 74, 046603 (2006).

[26] A. E. Miroshnichenko, S. Flach, and B. A. Malomed, Chaos 13, 874 (2003).

[27] S. F. Mingaleev, A. E. Miroshnichenko, and Yu. S. Kivshar, Opt. Express 15, 12380 (2007).

[28] A. E. Miroshnichenko, M. I. Molina, and Yu. S. Kivshar, Phys. Rev. E 75, 046602 (2007).

[29] T. White, C. de Sterke, R. McPhedran, T. Huang, and L. Botten, Opt. Express 12, 3035-3045 (2004).

[30] S. Fan, Appl. Phys. Lett. 80, 908 (2002).

[31] M. Fleischhauer, A. Imamoglu, and J. P. Marangos, Rev. Mod. Phys. 77, 633 (2005).

[32] S. F. Mingaleev, Yu. S. Kivshar, and R. A. Sammut, Phys. Rev. E 62, 5777 (2000). 